\title{
PROLIFERATION OF SMOOTH MUSCLE CELLS IN ACUTE ALLOGRAFT VASCULAR REJECTION
}

Jun Amano, MD

Shigeru Ishiyama, $\mathrm{MD}^{\mathrm{b}}$

Toshiro Nishikawa, MD ${ }^{\mathrm{b}}$

Hiroyuki Tanaka, MD $^{c}$

Ryozo Nagai, MD

Fumiaki Marumo, $\mathrm{MD}^{\mathrm{e}}$

Michiaki Hiroe, $\mathrm{MD}^{\mathrm{e}}$
Objectives: To determine whether immune injury during acute cardiac rejection induces phenotypic modulation of arterial smooth muscle cells and lesion formation, we studied the expression of embryonic myosin heavy-chain isoform and degrees of intimal proliferation in aortas and coronary arteries of allografted rabbit hearts. Modulation of phenotype in arterial smooth muscle cells during acute vascular injury is a widely reported phenomenon, and proliferation and migration of medial smooth muscle cells contribute to development of intimal hyperplasia of arteries in response to immune injury. Methods: Rabbit hearts were heterotopically transplanted to the neck without immunosuppression. Hearts were harvested at 2, 5, 7, and 10 days after transplantation. Proliferation of smooth muscle cells was assessed by bromodeoxyuridine labeling. Staining for immunohistochemical indicators was done with use of monoclonal antibodies that recognize $T$ lymphocytes and all types of smooth muscle cells (SM1), adult type of smooth muscle cells (SM2), and embryonic myosin heavy-chain isoform. Intimal thickening and luminal narrowing were assessed with a computer-assisted video image analysis system. Results: Intimal thickening and luminal narrowing in aortas and coronary arteries gradually increased in a time-dependent manner. The neointima thus formed consisted of proliferating smooth muscle cells positive for both SM1 and embryonic myosin heavy-chain isoform and massive $T$ lymphocyte accumulation. Intimal proliferation was more prominent in aortas and large epicardial coronary arteries than in the intramyocardial small coronary arteries. Conclusions: These findings suggest that allogeneic immune injury facilitates phenotypic modulation of smooth muscle cells, which may contribute to subsequent transplantation-associated atherosclerosis. (J Thorac Cardiovasc Surg 1997;113:19-25)
$T^{T}$ ransplantation-associated atherosclerosis remains a serious complication that limits the long-term success of cardiac transplantation. ${ }^{1-4}$ The pathophysiologic processes of this accelerated ath-

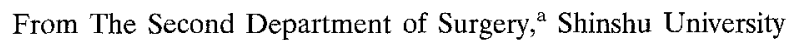
School of Medicine, Matsumoto, Nagano; Department of Cardiothoracic Surgery ${ }^{\mathrm{c}}$ and The Second Department of Medicine, ${ }^{\mathrm{e}}$ Tokyo Medical and Dental University; The Second Department of Pathology, Tokyo Women's Medical College, ${ }^{b}$ Tokyo; and The Second Department of Medicine, Gunma University, ${ }^{\mathrm{a}}$ Maebashi, Gunma, Japan.

Received for publication March 28, 1996; revisions requested May 13, 1996; revisions received June 6, 1996; accepted for publication June 11, 1996.

Address for reprints: Jun Amano, MD, The Second Department of Surgery, Shinshu University School of Medicine, 3-1-1 Asahi, Matsumoto, Nagano, 390 Japan.

Copyright (C) 1997 by Mosby-Year Book, Inc.

$0022-5223 / 97 \$ 5.00+0 \quad \mathbf{1 2 / 1 / 7 5 7 1 0}$ erosclerosis and the stimuli that induce it remain unclear. Whatever the initiating factors involved in this process, disordered control of smooth muscle cell (SMC) homeostasis in vessel walls appears likely to promote the intimal hyperplasia that produces obstructive lesions in vessels. ${ }^{5,6}$

Kuro-o and associates ${ }^{7}$ reported that rabbit SMCs express at least three types of myosin heavy-chain (MHC) isoforms: SM1, SM2, and SMemb. SM1 and SM2 are specific to SMCs, but SMemb is a nonmuscle-type MHC isoform. ${ }^{7,8} \mathrm{SM} 1$ is constitutively expressed from early development, but SM2 first appears after birth. SMemb is expressed predominantly in the embryonic and neonatal stages and disappears in adults. These previous studies have clearly demonstrated the importance of these MHC isoforms as molecular markers in the identification of abnormally proliferating SMCs in vascular lesion formation. 
It seems likely that in transplanted hearts, proliferation and migration of medial SMCs contribute to development of intimal hyperplasia of arteries in response to certain immune-mediated stimuli. ${ }^{5,6}$ Libby and Tanaka ${ }^{5}$ have presented evidence that the development of transplantation-associated arteriosclerosis involves a cellular immune reaction to alloantigens on graft arterial cells. Indeed, Tanaka and colleagues ${ }^{9,10}$ recently reported evidence for activation and proliferation of SMCs in coronary arteries during acute rejection as determined by the expression of leukocyte adhesion molecules and tumor necrosis factor- $\alpha$. To examine altered vascular SMC phenotypes in greater detail, we studied the expression of $\mathrm{MHC}$ isoforms and characteristics of neointimal lesions of both aortas and coronary arteries during acute rejection in allografted rabbit hearts.

\section{Material and methods}

Animals. Japanese white rabbits (Saitama Experimental Animal Lab., Saitama, Japan) were used as donors (1.5 to $2.0 \mathrm{~kg}$ ) and recipients $(2.5$ to $3.0 \mathrm{~kg}$ ). All animals were monitored regularly for accidental contamination with infectious diseases. They were fed standard commercial rabbit chow and water ad libitum and were handled humanely in accordance with the regulations of the Animal Care and Use Committee of the Tokyo Medical and Dental University.

Heart transplantation. The heterotopic heart transplantation procedure used in this study was a modification of the technique originally described by Carrel. ${ }^{11}$ The donor and recipient rabbits were anesthetized with intramuscular injections of ketamine $(35 \mathrm{mg} / \mathrm{kg})$ and xylazine ( $7 \mathrm{mg} / \mathrm{kg}$ ). A vertical incision was made on the anterior aspect of the neck of the recipient rabbit. The right common carotid artery and the external jugular vein were dissected free from surrounding tissues. Anticoagulation was achieved in the donor rabbit with an intravenous injection of heparin sodium (1000 IU). After thoracotomy, the donor heart was removed rapidly after ligation of the venae cavae and pulmonary veins and immersed in cold saline solution. The transected ascending aorta of the donor heart was anastomosed end-to-side to the common carotid artery of the recipient, and the pulmonary artery of the donor was anastomosed to the external jugular vein of the recipient. A minilaparotomy was then done to insert an osmotic pump (Alza Corp., Palo Alto, Calif.) for continuous infusion of bromodeoxyuridine $(20 \mathrm{mg} / \mathrm{kg}$ per day). The mean ischemic time, defined as the interval from removal of the graft to restoration of circulation, ranged from 30 to 40 minutes. The transplanted heart was examined daily by palpation. With the use of deep anesthesia (pentobarbital, $50 \mathrm{mg} / \mathrm{kg}$ ), the recipients were killed and their hearts excised at $2(n=7), 5(n=12), 7(n=$ $13)$, or $10(n=15)$ days after transplantation after intravenous injection of heparin sodium (1000 IU). Both the recipient and allograft hearts were perfused from the aorta with heparinized lactated Ringer's solution. Hearts were divided by three transverse sections at the basal, mid, and apical ventricular levels. Several longitudinal sections of coronary ostia including both the aorta and the heart were made. These sections of the heart and the aorta were either immersed fixed in ethanol acetate or frozen in isopentane-cooled liquid nitrogen and optimal cutting temperature compound (O. C. T. compound, Miles, Inc., Elkhart, Ind.).

General morphometry and histologic grading of rejection. All ethanol acetate-fixed tissues were embedded in paraffin, cut into $6 \mu \mathrm{m}$ sections, and stained with hematoxylin-eosin, Masson trichrome, and van Gieson elastin stains. Quantitative histologic analysis was done by two pathologists without knowledge of the protocol. Each section was examined for grade of myocardial rejection and the presence and extent of luminal narrowing and intimal thickening. Morphometric analyses were done on van Gieson elastin-stained segments with use of a computer-assisted color image analyzer system (SP500F, Olympus Inc., Tokyo). For these analyses, five large epicardial coronary arteries ( 3 to $4 \times 10 \mu \mathrm{m}$ in diameter) and five intramyocardial small coronary arteries $(10 \mu \mathrm{m}$ in diameter) were selected. Intimal, medial, and luminal areas and lesion thickness were measured. Percentage luminal narrowing $(\% \mathrm{LN})$ and ratio of intimal thickness (\%IT) were calculated with the following formulas: $\% \mathrm{LN}=$ intimal area/(intimal area + luminal area) $\times 100$ and $\% \mathrm{IT}=$ intimal thickness/(intimal thickness + medial thickness $) \times 100$. Myocardial rejection was graded with use of the classification of the International Society for Heart and Lung Transplantation standarized grading system on a scale of 1 to $4 .^{12}$

Immunohistochemistry. The following antibodies were used to differentiate cell types. For SMCs, anti-musclespecific actin antibody (HHF35, Enzo Biochem., New York, N.Y.) was used, and the expression of MHC isoforms was examined with anti-SM1, anti-SM2, and anti-SMemb antibodies as previously reported. ${ }^{7}$ For $T$ lymphocytes and macrophages, L-11/135 ${ }^{13}$ and RAM11 (Enzo Diagnostic Inc.) were used. Endothelial cells were identified with use of anti-von Willebrand factor and anti-human endothelial cell JC/70A (Dakopatts, Copenhagen).

Immunoperoxidase studies were done with the use of a modified version of previously described techniques. ${ }^{9}$ The sections were incubated overnight with antibodies and then the avidin-biotin complex immunoperoxidase procedure (Vectastain, Vector Lab. Inc., Burlingame, Calif.) was done. Antibody binding was visualized with 3,3'diaminobenzidine (Sigma Chemical Co., St. Louis, Mo.) and sections were counterstained with methyl green or hematoxylin.

The intensity and extent of staining of SMCs for SMemb and bromodeoxyuridine were graded semiquantitatively on a scale from 0 to 5 as follows: grade 0 , no staining; grade 1, scattered and infrequent staining; grade 2 , scattered mild staining; grade 3, scattered moderate staining; grade 4 , widespread staining; and grade 5 , widespread and intense staining.

Data analysis. All values are expressed as means plus or minus the standard deviation. Data were processed 

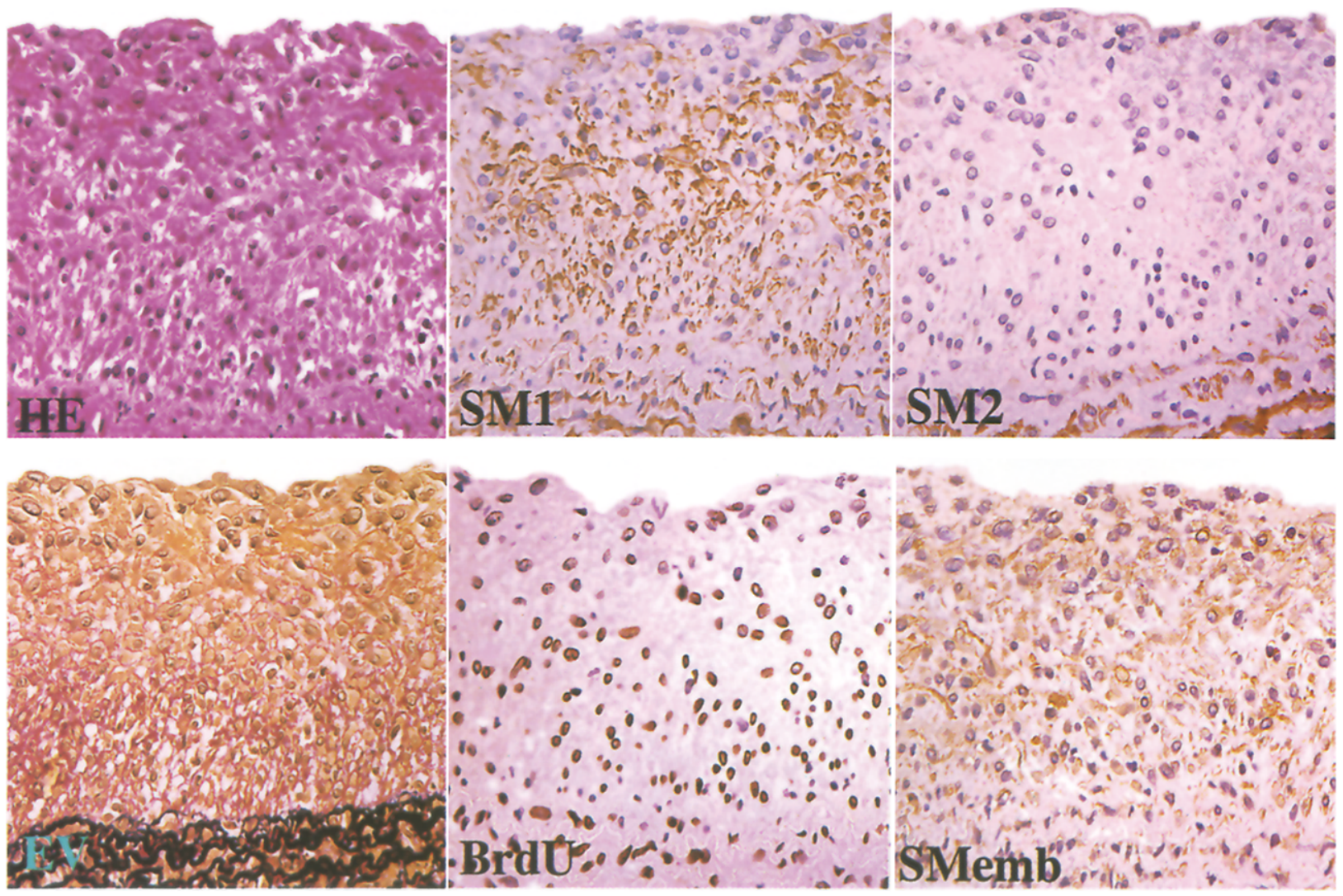

Fig. 1. Expression of MHC isoforms and bromodeoxyuridine ( $B r d U)$ in allografted aorta at 10 days after transplantation. Serial sections of ascending aorta of transplanted heart display severe intimal thickening. SM1-positive SMCs are negative for SM2, but positive for SMemb. $H E$, Hematoxylin-eosin stain; $E V$, van Gieson elastin stain (original magnification $\times 400$ ).

with StatView software (StatView-J 4.02, Brainpower Inc., Calabasas, Calif.). Significant differences among groups were determined by one-way analysis of variance and Scheffe's comparison method. Changes from control values were compared by the Mann-Whitney rank-sum test. Differences were considered significant when $p<0.05$.

\section{Results}

Grades of rejection in transplanted hearts. The recipient hearts exhibited no myocytic necrosis or cell infiltration. In the transplanted heart, there was minimal lymphocytic infiltrate around vessels without myocytic necrosis at 2 days after transplantation. At 5 days after transplantation, diffuse perivascular and interstitial cell infiltrates was present, and myocytic necrosis gradually increased thereafter. On days 7 and 10 after transplantation, severe rejection with widespread myocytic necrosis was present. The histologic degrees of rejection by the International Society for Heart and Lung Transplantation grading scale for allografts at $2,5,7$, and 10 days after transplantation were $1.6 \pm 0.5,2.8 \pm 0.7,3.4 \pm 0.5$, and $4 \pm 0.0$, respectively.

Histologic examination. None of the recipient aortas or coronary arteries exhibited any intimal thickening, and none expressed SMemb or incorporated bromodeoxyuridine. At 2 days after transplantation, there was also no intimal thickening or expression of SMemb in the SMCs in the donor aortas or coronary arteries. At 5 days after transplantation, intimal thickening mainly as a result of $\mathrm{T}$ cell accumulation was observed in the aorta. This type of intimal thickening was also observed in the large epicardial coronary artery. At 7 and 10 days after transplantation, intimal thickening of the aortas and the large coronary arteries became pronounced and circumferentially larger and was accompanied by widespread, extensive infiltration by mononuclear cells and cells with highly vacuolated 


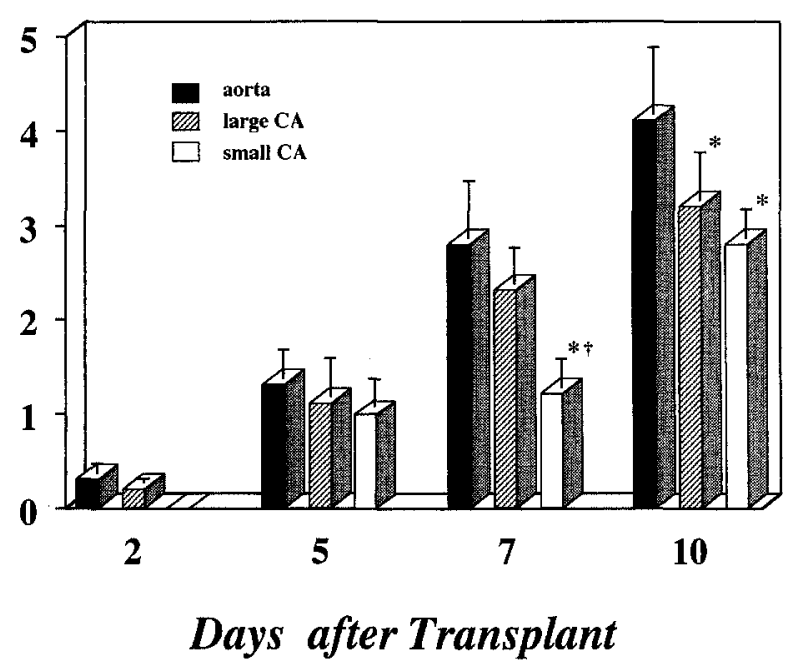

Fig. 2. Expression of bromodeoxyuridine in allografted aorta, epicardial large coronary arteries (large $C A$ ), and intramyocardial small coronary arteries (small $C A$ ) at 2, 5, 7 , and 10 days after transplantation. ${ }^{*} p<0.05$ compared with aorta; $t p<0.05$ compared with large coronary arteries.

cytoplasm. Most of the endothelial cells in the aorta were lost. These cells in the neointima were $T$ cells, and RAM11-positive macrophages were observed sporadically. The highly vacuolated cells in the neointima of the aortas and the large coronary arteries were negative for SM2, but expressed actin, SM1, and SMemb (Fig. 1). The cells positive for anti-SMemb were also stained with bromodeoxyuridine. There were fewer cells positive for SMemb and bromodeoxyuridine in the small coronary arteries than in the large coronary arteries. SMCs in arterioles did not express SMemb or incorporate bromodeoxyuridine. The degrees of expression of SMemb and bromodeoxyuridine in SMCs in the aorta, large epicardial coronary artery, and small intramyocardial coronary artery are illustrated in Figs. 2 and 3.

Morphometric analysis. In the aorta, percentages of both luminal narrowing and intimal thickness increased significantly over time from 2 days to 10 days after transplantation. At 7 days after transplantation, percentages of luminal narrowing and intimal thickness of the small intramyocardial coronary artery were significantly smaller than those for the large epicardial coronary artery (Table I, Fig. 4). In addition, at 10 days after transplantation, percentages of luminal narrowing and intimal thickness of the small intramyocardial coronary artery were

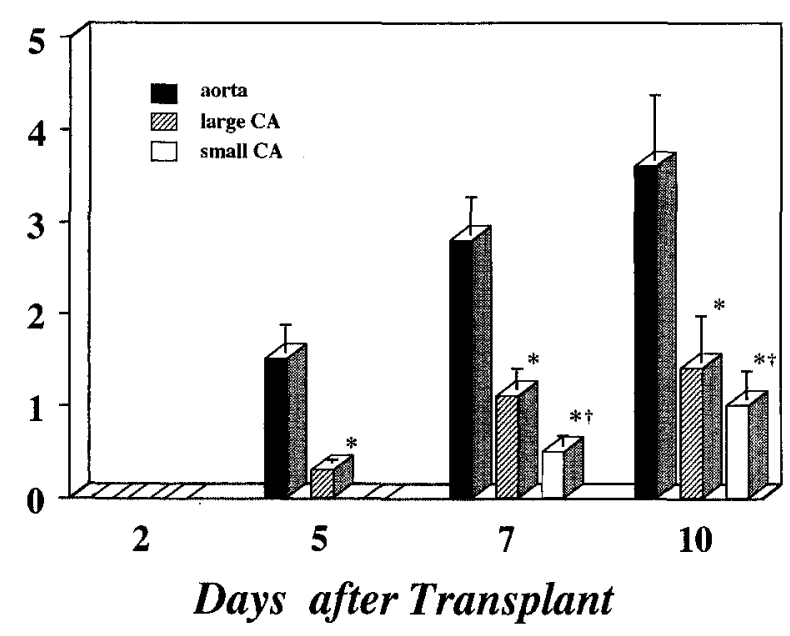

Fig. 3. Expression of SMemb in allografted aorta, epicardial large coronary arteries (large $C A$ ), and intramyocardial small coronary arteries (small $C A$ ) at $2,5,7$, and 10 days after transplantation. ${ }^{*} p<0.05$ compared with aorta; $\dagger p<0.05$ compared with large coronary arteries.

significantly smaller than those of the aorta and the large epicardial coronary artery (Table I, Fig. 4).

\section{Discussion}

The findings of previous experimental and clinical studies have suggested that immune-mediated stimuli induce transplantation-associated arteriosclerosis. ${ }^{4,5,9,10,14,15}$ However, clinical investigations disagree concerning the extent of correlation of the number of episodes or severity of acute rejection with the development of this disease. Tanaka and colleagues $^{9,10}$ recently reported activation and proliferation of vascular SMCs during acute rejection with use of the same rabbit heterotopic transplantation model as our own, suggesting that acute cell-mediated rejection might alter SMC phenotypes in rabbit arteries and induce SMC proliferation, thereby contributing to lesion formation. We therefore evaluated the expression of MHC isoforms of SMCs, which are molecular markers of phenotypic modulation of SMCs in arteries during acute rejection. In this study, we found expression of SMemb and decreased expression of SM2 in most neointimal SMCs and in addition found occasional medial SMCs in the aortas and the large coronary arteries with striking accumulation of $\mathrm{T}$ lymphocytes. These findings suggest that certain allogeneic immunemediated stimuli during acute rejection modulate MHC isoforms to induce migration and proliferation of SMCs. ${ }^{5,9,16}$ Possible candidates for inducers 
Table I. Morphometric data for aorta and coronary arteries

\begin{tabular}{|c|c|c|c|c|c|c|}
\hline & \multicolumn{3}{|c|}{7 Days } & \multicolumn{3}{|c|}{10 Days } \\
\hline & Aorta & Large $C A$ & Small $C A$ & Aorta & Large $C A$ & Small $C A$ \\
\hline External diameter $(\times 10 \mu \mathrm{m})$ & $336 \pm 48$ & $41 \pm 0.2$ & $10 \pm 0.07$ & $321 \pm 35$ & $42 \pm 0.3$ & $9.5 \pm 0.06$ \\
\hline \multicolumn{7}{|l|}{ Area } \\
\hline Medial $\left(\times 10^{5} \mu \mathrm{m}^{2}\right)$ & $44 \pm 5$ & $3.2 \pm 0.4$ & $0.41 \pm 0.05$ & $42 \pm 4$ & $3.3 \pm 0.6$ & $0.42 \pm 0.4$ \\
\hline Intimal $\left(\times 10^{3} \mu \mathrm{m}^{2}\right)$ & $298 \pm 31$ & $9.8 \pm 0.3$ & $0.64 \pm 0.05$ & $725 \pm 52$ & $13 \pm 0.4$ & $0.71 \pm 0.03$ \\
\hline Percent LN & $7.1 \pm 0.3$ & $22.4 \pm 6.5$ & $5.3 \pm 0.4^{*}$ & $17.5 \pm 0.4$ & $28.7 \pm 8.5$ & $9.5 \pm 0.6^{*} \dagger$ \\
\hline \multicolumn{7}{|l|}{ Thickness } \\
\hline Medial $(\times 10 \mu \mathrm{m})$ & $34 \pm 5$ & $4.3 \pm 0.2$ & $1.6 \pm 0.07$ & $35 \pm 6$ & $4.5 \pm 0.7$ & $1.5 \pm 0.05$ \\
\hline Intimal $(\mu \mathrm{m})$ & $4.1 \pm 0.5$ & $1.1 \pm 0.08$ & $0.17 \pm 0.02$ & $9.7 \pm 0.8$ & $2.9 \pm 0.3$ & $0.2 \pm 0.06$ \\
\hline Percent thickness & $10.5 \pm 0.8$ & $20.2 \pm 5.8$ & $8.9 \pm 0.7^{*}$ & $21.7 \pm 2.5$ & $39.7 \pm 6.7$ & $11.8 \pm 0.8^{*} \dagger$ \\
\hline
\end{tabular}

Values are means plus or minus the standard deviation. $C A$, Coronary artery; $L N$, luminal narrowing.

${ }^{*} p<0.05$ compared with large coronary artery.

$t p<0.05$ compared with aorta.

of isoform modulation of SMCs include cytokines and growth factors released from activated $\mathrm{T}$ lymphocytes that have infiltrated into vessels and from vascular endothelial cells and SMCs themselves. ${ }^{5,9,16}$

Differential expression of MHC. Another issue concerning expression of $\mathrm{MHC}$ isoforms in this study is why facilitated immature phenotype expression of SMCs was less pronounced in the coronary artery than in the aorta. We suspect that a differentiation or modulation process may affect the heterogenous formation of immature type of SMCs. In support of this, Tanaka and colleagues ${ }^{9,10}$ reported that heterogenous activation of endothelial cells and SMCs, as determined by the expression of vascular cell adhesion molecule 1, intracellular adhesion molecule 1 , and tumor necrosis factor- $\alpha$, was more rapid and more pronounced in the aorta than in the coronary artery during acute rejection in rabbit allograft hearts. In contrast, the possibility exists that normal adult rabbit arterial media is composed of phenotypically heterogenous SMC populations or stemlike cell compartments that increase in size to perform various functions in response to different stimuli. ${ }^{17,18}$ Zanellato and coworkers ${ }^{17}$ identified two SMC populations in the medial layer of normal adult rabbit aorta: cells that contained smooth muscle myosin exclusively and cells with both smooth muscle and nonmuscle myosin $(200 \mathrm{kDa})$ isoforms. The size of the cell population with double myosin isoform content increased markedly over the course of experimental atherogenesis. Frid, Moiseeva, and Stenmark ${ }^{18}$ also obtained evidence that suggested the presence of complex, site-specific heterogeneity in the structure and cellular composition of the bovine main pulmonary artery. They reported the presence of an abundant nonmuscle-like cell or immature SMC population within the mature arterial media. Given these observations of SMC heterogeneity in mature vessels and the findings of the present study, it seems reasonable to speculate that a specific immature type of SMC subpopulation that is not labeled by anti-SMemb antibody but expresses other nonmuscle myosin isoforms may be more abundant in the aorta than in the coronary artery. In fact, a difference exists in the expression of SMemb between human and rabbit vascular $\mathrm{MHC}^{19}$ In rabbits, SMemb quickly disappeared at 4 months after birth, whereas in human beings, SMemb was detected even in adults in aorta and coronary arteries. However, smaller-sized arteries, including the vasa vasorum of the aorta or intramyocardial coronary arteries, were negative for SMemb. ${ }^{19}$ Thus further analysis may be required to determine the relevance of the findings of the present study to human cardiac transplantation.

Differential intimal thickening during acute rejection in various types of arteries. Our finding of greater annular intimal thickening in the epicardial large coronary artery than in the intramyocardial small coronary artery is quite different from those findings described in previous studies of chronic transplantation-associated arteriosclerosis. ${ }^{1-3,20}$ Johnson and associates $^{2,3}$ reported that transplant coronary artery disease of long-term allografts is often diffuse, especially in distal vessels. Lin and colleagues ${ }^{21}$ also found that percentage luminal narrowing in human transplant recipients did not differ between proximal and distal segments in the left anterior descending coronary arteries ( $46 \%$ proximally versus $41 \%$ distally) in a study of 25 allografts at autopsy and explants at a mean of 490 days after transplantation. Disparity in time of 

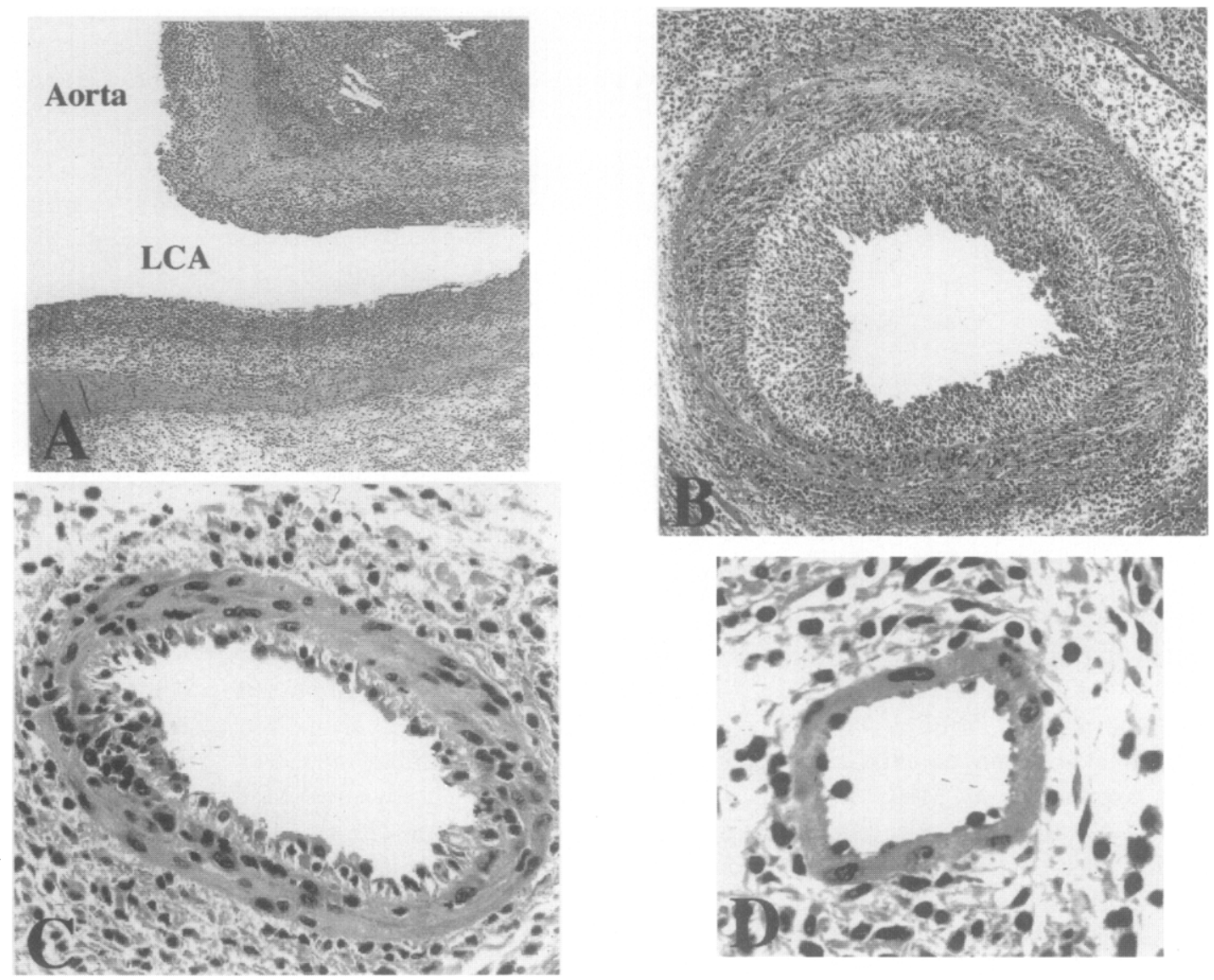

Fig. 4. Sections of aorta and coronary arteries in allografted heart at 10 days after transplantation stained with hematoxylin-eosin stain. A, Longitudinal section of aorta and ostium of left coronary artery ( $L C A)$ (original magnification $\times 40$ ); $\mathbf{B}$, cross-section of proximal left anterior descending coronary artery (original magnification $\times 100$ ); $\mathbf{C}$, cross-section of intramyocardial small coronary artery (original magnification $\times 400$ ); D, cross-section of arteriole (original magnification $\times 400$ ).

study after transplantation, and also differences among clinical human transplantation and experimental models with or without hyperlipidemia, may have accounted for the differences in results obtained. In addition, the lack of immunosuppression compared with other animal models that examined the development of atherosclerosis may explain the obtained different results. However, several reports have suggested that proliferative intimal lesions during acute vascular rejection are not caused by accelerated atherogenesis, but represent a separate disease entity in which infiltrating $\mathrm{T}$ lymphocytes may play a critical role in initiating intimal SMC accumulation and proliferation. ${ }^{4,22-24}$ Norman and associates ${ }^{23}$ demonstrated proliferation of vacuolated immature SMCs in the neointima accompanied by mainly $\mathrm{T}$ lymphocyte infiltration during acute rejection in four transplanted human hearts surviving 10 to 82 days. They also reported that acute vascular rejection was present in the main coronary arteries and the major epicardial branches, but was usually not observed in the intramyocardial vessels. In the present study, we also found that highly vacuolated cells were present in the neointima of the aortas and the large coronary arteries that express SMemb. Accordingly, it seems likely that acute vascular rejection occurred predominantly in large coronary arteries, whereas chronic transplantation-associated atherosclerosis occurred diffusely or was more pronounced in small coronary arteries. Although it is difficult to clarify in what fashion acute annular lymphocyte accumulation changes to the formation of the typical chronic myointimal lesions, and these two lesions are probably different stages in 
the natural history of immune-mediated vascular injury, acute vascular rejection may be pathologically distinct from chronic vascular rejection.

Conclusion. The findings of the present study elucidate one aspect of the pathogenesis of graft atherosclerosis: that allogeneic immune injury facilitates phenotypic modulation of SMCs, which may contribute to subsequent transplantation-associated atherosclerosis. However, the questions of how episodes of acute rejection affect the development of late-stage transplantation-associated graft atherosclerosis and why heterogeneity of intimal lesion is present among arteries remain to be answered.

We acknowledge Toshiyuki Maruyama, MD, for his consultation regarding statistical analysis.

\section{REFERENCES}

1. Chomette G, Auriol M, Cabrol C. Chronic rejection in human heart transplantation. J Heart Transplant 1988;7: 292-7.

2. Johnson DE, Gao S, Schoeder JS, DeCampli WM, Billingham ME. The spectrum of coronary artery pathologic findings in human cardiac allografts. J Heart Transplant 1987;8: 349-59.

3. Johnson DE, Alderman E, Schroeder JS, et al. Transplant coronary artery disease: histopathologic correlations with angiographic morphology. J Am Coll Cardiol 1991;17:449-57.

4. Norman SJ, Salomon DR, Leelachaikul P, et al. Acute vascular rejection of the coronary arteries in human heart transplantation: pathology and correlations with immunosuppression and cytomegalovirus infection. $\mathbf{J}$ Heart Lung Transplant 1991;10:674-87.

5. Libby $\mathrm{P}$, Tanaka $\mathrm{H}$. The pathogenesis of coronary arteriosclerosis ("chronic rejection") in transplanted hearts. Clin Transplant 1994;8:313-8.

6. Sanders M. Molecular and cellular concepts in atherosclerosis. Pharmacol Ther 1994;61:109-53.

7. Kuro-o M, Nagai R, Nakahara K, et al. cDNA cloning of a myosin heavy chain isoform in embryonic smooth muscle and its expression during vascular development and in arteriosclerosis. J Biol Chem 1991;266:3768-73.

8. Okamoto E, Imataka K, Fujii J, et al. Heterogeneity in smooth muscle cell population accumulation in the neointimas and the media of poststenotic dilatation of the rabbit carotid artery. Biochem Biophys Res Commun 1991;266: 3768-73.

9. Tanaka H, Sukhova G, Swanson SC, Cybulsky MI, Schoen FJ, Libby P. Endothelial and smooth muscle cells express leukocyte adhesion molecules heterogenously during acute rejection of rabbit cardiac allografts. Am J Pathol 1994;144: 938-51.
10. Tanaka H, Suzuki A, Sukhova G, Schoen FJ, Libby P. Expression of tumor necrosis factor- $\alpha$ in medial smooth muscle cells in coronary arteries during acute rejection of rabbit cardiac allografts (abstract). J Heart Lung Transplant 1994;13:S93.

11. Carrel A. The surgery of blood vessels. Bull Johns Hopkins Hosp 1907;18:18-28.

12. Billingham ME, Cary NR, Hammond ME, et al. A working formulation for the standardization of nomenclature in the diagnosis of heart and lung rejection. J Heart Transplant 1990;9:587-93.

13. Kume N, Cybulsky MI, Gimbrone MA Jr. Lysophosphatidylcholine, a component of atherogenic lipoproteins, induces mononuclear leukocyte adhesion molecules in cultured human and rabbit arterial endothelial cells. J Clin Invest 1992;90:1138-44.

14. Hruban RH, Beschorner WE, Baumgartner WA, et al. Accelerated arteriosclerosis in heart transplant recipients is associated with a T-lymphocyte-mediated endothelialitis. Am J Pathol 1990;137:871-82.

15. Kuwahara M, Jacobsson J, Kuwahara M, Kagan E, Ramwell PW, Foegh ML. Coronary artery ultrastructure changes in cardiac transplant atherosclerosis in the rabbit. Transplantation 1991;52:759-65.

16. Page C, Rose M, Yacoub M, Pigott R. Antigenic heterogeneity of vascular endothelium. Am J Pathol 1992;141:673-83.

17. Zanellato AMC, Borrione AC, Tonello M, Scannapieco G, Pauletto P, Sartore S. Myosin isoform expression and smooth muscle cell heterogeneity in normal and atherosclerotic rabbit aorta. Arteriosclerosis 1990;10:996-1009.

18. Frid MG, Moiseeva EP, Stenmark KR. Multiple phenotypically distinct smooth muscle cell populations exist in the adult and developing bovine pulmonary arterial media in vivo. Circ Res 1994;75:669-81.

19. Aikawa M, Sivam PN, Kuro-o M, et al. Human smooth muscle myosin heavy chain isoforms as molecular markers for vascular development and atherosclerosis. Circ Res 1993;73: 1000-12.

20. Billigham M. Cardiac transplant atherosclerosis. Transplant Proc 1987;119(suppl 5):19-25.

21. Lin H, Wilson JE, Kendall TJ, et al. Comparable proximal and distal severity of intimal thickening and size of epicardial coronary arteries in transplant arteriopathy of human cardiac allografts. J Heart Lung Transplant 1994;13:824-33.

22. Frazier OH, McAllister HA, Jammal CT, et al. Occlusive coronary arteritis: a cause of early death in a cardiac transplant patient. Ann Thorac Surg 1987;43:554-6.

23. Norman SJ, Khan SR, Leelachaikul P, Salomon DR. Origin of cells in the coronary intima during acute vascular rejection of the transplanted human heart. J Heart Lung Transplant 1992;11:492-9.

24. Yowell RL, Hammond EH, Bristow MR, Watson FS, Renlund DG, O'Connell JB. Acute vascular rejection involving the major coronary arteries of a cardiac allograft. J Heart Transplant 1988;7:191-7. 